\title{
Effects of Acute Exercise and Chronic Exercise on the Liver Leptin-AMPK-ACC Signaling Pathway in Rats with Type 2 Diabetes
}

\author{
Xuejie Yi, ${ }^{1,2}$ Shicheng Cao, ${ }^{3}$ Bo Chang, ${ }^{2}$ Dalin Zhao, ${ }^{2}$ Haining Gao, \\ Yihan Wan, ${ }^{2}$ Jiaojiao Shi, ${ }^{2}$ Wei Wei, ${ }^{2}$ and Yifu Guan ${ }^{1}$ \\ ${ }^{1}$ Department of Biochemistry and Molecular Biology, College of Basic Medical Sciences, China Medical University, \\ Shenyang 110001, China \\ ${ }^{2}$ Department of Exercise Science, Shenyang Sport University, Shenyang, China \\ ${ }^{3}$ Department of Sport Medicine, College of Basic Medical Sciences, China Medical University, Shenyang, China
}

Correspondence should be addressed to Yifu Guan; yfguan@mail.cmu.edu.cn

Received 13 August 2013; Revised 1 November 2013; Accepted 1 November 2013

Academic Editor: Giuseppe Paolisso

Copyright (C) 2013 Xuejie Yi et al. This is an open access article distributed under the Creative Commons Attribution License, which permits unrestricted use, distribution, and reproduction in any medium, provided the original work is properly cited.

\begin{abstract}
Aim. To investigate the effects of acute and chronic exercise on glucose and lipid metabolism in liver of rats with type 2 diabetes caused by a high fat diet and low dose streptozotocin (STZ). Methods. Animals were classified into control (CON), diabetes (DC), diabetic chronic exercise (DCE), and diabetic acute exercise (DAE) groups. Results. Compared to CON, the leptin levels in serum and liver and ACC phosphorylation were significantly higher in DC, but the levels of liver leptin receptor, AMPK $\alpha 1 / 2, A M P K \alpha 1$, and ACC proteins expression and phosphorylation were significantly lower in DC. In addition, the levels of liver glycogen reduced significantly, and the levels of TG and FFA increased significantly in DC compared to CON. Compared to DC, the levels of liver AMPK $\alpha 1 / 2$, AMPK $\alpha 2$, AMPK $\alpha 1$, and ACC phosphorylation significantly increased in DCE and DAE. However, significant increase of the level of liver leptin receptor and glycogen as well as significant decrease of the level of TG and FFA were observed only in DEC. Conclusion. Our study demonstrated that both acute and chronic exercise indirectly activated the leptin-AMPK-ACC signaling pathway and increased insulin sensitivity in the liver of type 2 diabetic rats. However, only chronic and long-term exercise improved glucose and lipid metabolism of the liver.
\end{abstract}

\section{Introduction}

Leptin deficiency or dysfunction is one of the main causes for insulin resistance (IR) and lipid metabolism disorders $[1,2]$. However, patients with type 2 diabetes rarely have a leptin deficiency. It has been found that the majority of type 2 diabetes patients have higher levels of body fat, but normal or increased leptin in the plasma [3-6], indicating leptin resistance (LR). Certain levels of leptin effectively could stimulate AMP-activated protein kinase (AMPK) to phosphorylate acetyl-coA carboxylase (ACC), which in turn reduces the ACC activity, decreases fatty acid synthesis [7], and increases the oxidation of fatty acid (FA) [8], consequently, maintaining the balance of lipid metabolism in the body. Studies have shown that even one week of a high fat diet can cause leptin to increase rapidly, leading to fat accumulation in peripheral tissue IR [9]. Obese persons with high serum leptin levels tend to experience a downregulation of leptin receptor in hypothalamus, adipose tissue, and liver [10], which causes peripheral tissues to become LR and promotes lipid accumulation [11-13]. Excessive lipid deposition in nonfat tissue has been known to have a toxic effect on cells and to reduce sensitivity to insulin, eventually leading to diabetes and metabolic syndrome [14]. Liver plays an important role in the regulation of glucose metabolism and lipid metabolism. Moreover, type 2 diabetes and liver steatosis often coexist [15-17], but the causal mechanism is unclear. The possible trigger for type 2 diabetes might be related to leptin resistance, which further inhibits the liver AMPK-ACC signaling pathway and causes liver and systemic metabolic disorders. 
Exercise can reduce body fat by increasing energy consumption and improve the leptin resistance [18, 19]. However, how the leptin resistance is improved remains unclear. Previous studies have shown that exercise could upregulate the expression of leptin receptor and induce changes of the JAK-STAT3 signaling pathway in the hypothalamus and peripheral tissues in leptin-resistant rats [20, 21]. After exercise, normal rats experienced increased skeletal muscle growth, increased activity of the liver AMPK and MalonylCoA decarboxylase (MCD) pathways, and decreased activity of ACC, which further increased fatty acid oxidation and reduced glyceride synthesis [22]. Our previous study suggested that chronic and acute exercise could both reduce obesity and decrease blood sugar level in type 2 diabetic rats as well as improve the phosphorylation and gene expression related to improved skeletal muscle; $\mathrm{AMPK} \alpha 1 / 2$ and its subunits (AMPK $\alpha 1, A M P K \alpha 2)$ reduced ACC phosphorylation [23].

It is unknown, however, whether exercise affects the liver leptin-AMPK signaling pathway or whether it can improve liver lipid metabolism. In our present study, a type 2 diabetic rat model, given a high fat diet and a low dose of STZ, was used to address this question. Acute and chronic exercise variables were chosen to study whether exercise could affect protein expression and protein phosphorylation involved in the liver leptin-AMPK-ACC signaling pathway. The relationship between the liver leptin-AMPK-ACC signaling pathway and lipid metabolism, as well as the effect of acute and chronic exercise on that pathway, was investigated.

\section{Material and Methods}

2.1. Animals. 15-month-old male SD rats (450-470 g) were provided by the Animal Center of the Academy of Military Medical Sciences of the Chinese People's Liberation Army (certification number SCXK (army) 2007-004). Animals were housed under standard conditions $\left(22 \pm 2^{\circ} \mathrm{C}\right.$, humidity $50 \pm$ $10 \%$, cycles of $12 \mathrm{~h}$ light $/ 12 \mathrm{~h}$ dark). Experimental procedures were performed in accordance with the Guidance Suggestions for the Care and Use of Laboratory Animals, formulated by the Ministry of Science and Technology of the People's Republic of China in 1998, and were approved by the Animal Ethics Committee of China Medical University.

2.2. Animal Models. Rats were randomly divided into a control diet group (CON) and a high fat diet group (HFD). The control diet contained $57.3 \%$ carbohydrates, $18.1 \%$ protein, $18.8 \%$ cellulose, and $4.5 \%$ fat and the high fat diet consisted of $23 \%$ soy protein, $19.8 \%$ pork fat, $19.8 \%$ corn oil, $24.5 \%$ sucrose, and $5 \%$ cellulose and was supplemented with a $1.4 \%$ vitamin mixture, $6.7 \%$ mineral mixture, and $0.2 \%$ choline bitartrate. After 8 weeks, HFD rats were administered $30 \mathrm{mg} / \mathrm{kg}$ STZ (citrated buffer, $\mathrm{pH} 4.4$, Sigma) by intraperitoneal injection, while CON rats were injected with the same volume of citrate buffer $(1 \mathrm{~mL} / \mathrm{kg})$. Four weeks after injection with STZ, all animals with fasting blood above $7.8 \mathrm{mmol} / \mathrm{L}$ and postprandial glucose above $11.1 \mathrm{mmol} / \mathrm{L}$ were considered to be diabetic $[24,25]$. Diabetic rats were then divided into a diabetic control group (DC), diabetic chronic exercise group (DCE), and diabetic acute exercise (DAE). Body weight, water, and food intake were examined every week.

2.3. Chronic Exercise. Rats were trained to swim 10-20 min per session for more than 2 days to reduce water-induced stress. Two or three rats per group were placed in a plastic cylindrical pool of $45 \mathrm{~cm}$ in diameter and $60 \mathrm{~cm}$ of deep, with a water temperature of $34-35^{\circ} \mathrm{C}$. After the initial training, the rats underwent chronic exercise for 1 hour/day, 5 days/week, for 8 weeks. The exercise program was conducted in accordance with the Luciano E program with some modifications [26].

2.4. Acute Exercise. Acute exercise was conducted in two 90-minute sessions with a 45-minute interval between each session. The exercise program was performed in accordance with the Chibalin AV program with some modification [27]. Animal model preparation and exercise sessions were conducted in the Shenyang Institute of Physical Education Laboratory.

2.5. Blood Samples Collection and Blood Biochemistry. 2436 hours after the final session of chronic exercise or 8-16 hours after acute exercise, all rats were anesthetized by single dose intraperitoneal injection of amobarbital $(15 \mathrm{mg} / \mathrm{kg})$. Blood samples were collected from tail veins and serum was separated by centrifugation at $1100 \times \mathrm{g}$ for $10 \mathrm{~min}$. Serum glucose, triglyceride, total cholesterol, free fatty acid, HDLC, LDL-C, and serum leptin levels were measured using an AutoAnalyzer (RT-1904C; Rayto, China). Serum insulin concentration was determined using a radioimmunoassay described by Laemmli [28].

2.6. Insulin Tolerance Test (ITT) and Serum Insulin Quantification. Insulin tolerance tests were performed after blood sample collection. The rats fasted for 12 hours were anesthetized and given $1.5 \mathrm{IU} / \mathrm{kg}$ of synthetic insulin (Sigma). Blood samples were collected at $0,5,10,15,20,25$, and $30 \mathrm{~min}$ after injection, centrifuged at $1100 \times \mathrm{g}$ for $15 \mathrm{~min}$ at $4^{\circ} \mathrm{C}$, and stored at $-20^{\circ} \mathrm{C}$ to determine glucose concentrations. The plasma glucose $\left(t_{1 / 2}\right)$ was calculated from the slope of the last square fit of the plasma glucose concentration during the linear phase of decline [26]. Serum insulin was also measured.

2.7. Liver Sampling. After ITT, the animals were sacrificed under anesthesia by intraperitoneal injection of sodium thiopental $(200 \mathrm{mg} / \mathrm{kg}$, following the recommendations of the US National Institutes of Health). The liver was isolated and placed in liquid nitrogen and then immediately transferred to $-80^{\circ} \mathrm{C}$. Western blotting analysis and the detection of liver glycogen, FAA, and TG were performed later.

2.8. Liver Glycogen Content. The frozen livers were weighed, digested with $1 \mathrm{~mol} / \mathrm{L} \mathrm{NaOH}(1: 9 \mathrm{wt} / \mathrm{vol})$ at $80^{\circ} \mathrm{C}$ for $10 \mathrm{~min}$, neutralized with $1 \mathrm{~mol} / \mathrm{L} \mathrm{HCl}$, and mixed with $6 \mathrm{~mol} / \mathrm{L} \mathrm{HCl}$ to a final concentration of $2 \mathrm{~mol} / \mathrm{L} \mathrm{HCl}$. The resulting solution was incubated at $85^{\circ} \mathrm{C}$ for 2 hours and neutralized again with 
TABLE 1: Body weight and biochemical parameters of different groups.

\begin{tabular}{lcccc}
\hline & CON & DC & DCE & DAE \\
\hline Weight (g) & $520.7 \pm 12.00$ & $502.8 \pm 10.51$ & $527.3 \pm 10.77$ & $507.3 \pm 13.16$ \\
Epididymal fat $(\mathrm{g})$ & $8.83 \pm 0.43$ & $7.86 \pm 0.34$ & $8.61 \pm 0.44$ & $8.12 \pm 0.45$ \\
Insulin (pmol/L) & $66.14 \pm 2.80$ & $76.29 \pm 4.17$ & $70.86 \pm 3.61$ & $68.70 \pm 3.34$ \\
FBG (mmol/L) & $5.35 \pm 0.23$ & $13.18 \pm 0.52^{* *}$ & $11.57 \pm 0.42^{\#}$ & $11.51 \pm 0.41^{\#}$ \\
Kitt (\%/min) & $3.19 \pm 0.19$ & $1.95 \pm 0.30^{* *}$ & $2.87 \pm 0.22^{\#}$ & $2.61 \pm 0.15^{\#}$ \\
TG (mmol/L) & $1.68 \pm 0.09$ & $2.06 \pm 0.11^{* *}$ & $1.79 \pm 0.10^{\#}$ & $2.12 \pm 0.07^{\$}$ \\
TC (mmol/L) & $4.15 \pm 0.26$ & $5.78 \pm 0.39^{* *}$ & $0.69 \pm 0.21^{\#}$ & $5.67 \pm 0.35^{\$}$ \\
FFA (mmol/L) & $0.39 \pm 0.01$ & $0.63 \pm 0.05^{* *}$ & $1.358 \pm 0.04^{\#}$ & $0.16^{\#}$ \\
HDL-C (mmol/L) & $1.730 \pm 0.33 \pm 0.04$ \\
LDL-C (mmol/L) & $0.27 \pm 0.12$ & $1.029 \pm 0.10^{* *}$ & $0.51 \pm 0.19^{\# \#}$ & $0.94 \pm 0.29$ \\
Leptin (ng/mL) & $3.25 \pm 0.38$ & $1.21 \pm 0.24^{* *}$ & $4.41 \pm 0.61^{\#}$ & $1.25 \pm 0.35^{\$ \$}$ \\
\hline
\end{tabular}

Results were expressed as mean \pm standard error $(n=7-8)$. Differences between groups were compared by one-way analysis of variance (ANOVA).

Note: in comparison with the control group: ${ }^{* *} P<0.01,{ }^{*} P<0.05$; in comparison with the diabetes group: ${ }^{\# \#} P<0.01,{ }^{\#} P<0.05$; in comparison with the chronic exercise group: ${ }^{\$ \$} P<0.01,{ }^{\$} P<0.05$.

$5 \mathrm{~mol} / \mathrm{L} \mathrm{NaOH}$ [29]. A glucose hexokinase assay kit (Sigma) was used to determine the concentration of hydrolyzed glucose and glucose content was determined as micromolar per gram of tissue. Liver FFA was determined with fatty acid kit (Sigma) following the manufacturer's instructions. The liver TG was determined with triglyceride determination kit (Sigma) following the manufacturer's instructions [28].

2.9. Tissue Extraction and Western Blotting. The frozen liver was thawed, weighed, roughly cut, placed in protein extraction solution (1\% Triton X-100, $100 \mathrm{mM}$ Tris, $\mathrm{pH} 7.4$, containing $100 \mathrm{mM}$ sodium pyrophosphate, $100 \mathrm{mM}$ sodium fluoride, $10 \mathrm{mM}$ ethylenediaminetetraacetic acid, $10 \mathrm{mM}$ sodium vanadate, $2 \mathrm{mM}$ phenylmethyl sulfonylfluoride, and $0.1 \mathrm{mg} / \mathrm{mL}$ aprotinin), and ultrasonicated at maximum speed at $4^{\circ} \mathrm{C}$ for $30 \mathrm{~s}$ (JY92-IIN; Scientz, Ningbo, China). The homogenate was centrifuged at $9000 \times \mathrm{g}$ at $4^{\circ} \mathrm{C}$ for $40 \mathrm{~min}$ (HC-3618R; Zonkia, Hefei, China). Nonsoluble material was discarded. The protein concentration in the supernatant was quantified using Bradford's method. Then, $100 \mu \mathrm{g}$ of tissue extract was mixed with an equal volume of $3 \times$ sample buffer solution (6.86 M urea, 4.29\% SDS, $300 \mathrm{mM}$ DTT, and $43 \mathrm{mM}$ Tris $\cdot \mathrm{HCl}, \mathrm{pH} 6.8$ ) at room temperature for $30 \mathrm{~min}$, subjected to sodium dodecyl sulfate-polyacrylamide gel electrophoresis (SDS-PAGE, 10\% polyacrylamide gels), and transferred to a polyvinylidene difluoride membrane at $4^{\circ} \mathrm{C}$ for $2 \mathrm{~h}$. The membrane was blocked using trihydroxymethyl aminomethane buffer salt + Tween-20 (TBST) containing $5 \%$ bovine serum albumin (Sigma) and washed with TBST ( $\mathrm{pH}$ 7.4). The antibody was dissolved in TBST containing $1 \%$ bovine serum albumin overnight at $4^{\circ} \mathrm{C}$. The used antibodies include leptin, leptin receptors, phosphorylated (p)AMPK $\alpha 1\left(\mathrm{Th}^{172}\right)$, AMPK $\alpha 1$, p-AMPK $\alpha 2\left(\mathrm{Thr}^{172}\right), \mathrm{AMPK} \alpha 2$, p-AMPK $\alpha 1 / 2\left(\mathrm{Thr}^{172}\right)$, AMPK $\alpha 1 / 2$, acetyl-CoA carboxylase (ACC), and p-ACC $\left(\mathrm{Ser}^{79}\right.$ ) (Cell Signaling Technology, Beverly, MA, USA; 1:1000 dilution). Bands of interest were visualized by enhanced chemiluminescence and absorbance was determined using FluorChem V2.0 gel imaging analysis software (Alpha Innotech, San Leandro, CA, USA).
2.10. Statistical Analysis. Results were presented as mean \pm standard error (SE). Differences between groups were compared by one-way analysis of variance (ANOVA). Values of $P<0.05$ were considered statistically significant. Statistical analyses were performed using JMP software (SAS Institute, Cary, NC).

\section{Results}

Table 1 reports the body weights and biochemical parameters of different groups of rats. When comparing with the control group, the DC group shows a significant increase of the concentrations of blood glucose, leptin, triglyceride, total cholesterol, free fatty, acid and LDL-C. The DC group also shows the decreased insulin sensitivity and HDL-C level, respectively. On the other hand, the body weight, epididymal fat mass, and blood insulin concentration exhibit small variations. All these changes are the typical metabolism characteristics of type II diabetes, proving that the animal model prepared using high fat diet plus STZ-induced disorders is suitable for the current study.

Data in Table 1 also illustrates the effect of chronic exercise on the DC rats. Chronic exercise could significantly reduce the blood glucose, leptin, triglycerides, total cholesterol, free fatty acids, and LDL-C. In contrast, chronic exercise could also increase insulin sensitivity and HDL-C levels. The acute exercise reduces blood glucose but increases the blood glucose disappearance rate and there were no significant effects on dyslipidemia and plasma leptin concentrations.

Compared to the CON group, liver glycogen of the DC group significantly decreased, but TG and FFA levels were significantly elevated (Table 2). This indicates a disorder of the liver glycolipid reserves and FAA. As shown in Table 2, chronic exercise effectively increased glycogen content and reduced liver TG and FAA, whereas acute exercise seemed to have no effect on the liver glucose and lipid reserves.

To understand the underlying mechanisms of the different exercises on the type 2 diabetes rats, the protein expression and protein phosphorylation profiles were examined 
TABLE 2: The effects of exercise on liver glycolipid storage in type 2 diabetes rats.

\begin{tabular}{lcccc}
\hline & CON & DC & DCE & DAE \\
\hline Hepatic G $(\mathrm{mg} / \mathrm{g})$ & $42.31 \pm 5.80$ & $19.86 \pm 2.32^{*}$ & $38.45 \pm 6.34^{\#}$ & $17.64 \pm 4.67$ \\
Hepatic TG $(\mu \mathrm{mol} / \mathrm{g})$ & $35.2 \pm 20.1$ & $144.1 \pm 50.4^{* *}$ & $51.0 \pm 15.3^{\# \#}$ & $138.4 \pm 33.4$ \\
Hepatic FAA $(\mu \mathrm{mol} / \mathrm{g})$ & $103.4 \pm 35.8$ & $179.4 \pm 36.2^{* *}$ & $116.9 \pm 27.5^{\#}$ & $163.9 \pm 43.9$ \\
\hline
\end{tabular}

Results were expressed as mean \pm standard error $(n=7-8)$. Differences between the groups were compared by one-way analysis of variance (ANOVA). Note: compared with normal control group: ${ }^{* *} P<0.01,{ }^{*} P<0.05$; compared with the diabetes control group: ${ }^{\# \#} P<0.01,{ }^{\#} P<0.05$.

using western blotting (Figure 2) and these image data were quantified with gel analysis software and presented in histograph (Figure 1). The DC group had a significantly increased expression of leptin with respect to that of the control group. The leptin expression returned to the basal level after either chronic or acute exercise (Figure 1(a)). However, the expression of leptin receptor showed an opposite change: a relatively low level of leptin receptor for the DC group and a restored level for the chronic exercise (DCE) and acute exercise (DAE) groups, respectively (Figure 1(a)). Interestingly, the ACC expression exhibited a similar trend to that of leptin, and the phosphorylated ACC exhibited a similar trend to that of leptin receptor (Figure 1(e)).

AMP-activated protein kinase (AMPK) is a kinase responsible for the downstream protein phosphorylation. It is composed of two subunits, AMPK $\alpha 1$ and AMPK $\alpha 2$. Previous studies have identified several phosphorylation positions on each subunit, and the phosphorylation could occur separately or simultaneously. The most frequently observed phosphorylation position is $\mathrm{Thr}^{172}$.

As shown, protein expression and the phosphorylation levels of $\mathrm{AMPK} \alpha 1 / 2, \mathrm{AMPK} \alpha 1$, and AMPK $\alpha 2$ were greatly reduced in the DC group (Figures 2(b), 2(c), and 2(d)). After chronic exercise, the protein expression and the protein phosphorylation of AMPK $\alpha 1 / 2$, AMPK $\alpha 1$, and AMPK $\alpha 2$ were elevated back to the normal level in both DCE and DAE groups.

The ACC phosphorylation was reduced in the DC group compared to the control group, which is consistent with the increased liver TG and FFA levels shown in Table 2. It suggests that the disruption of the leptin-AMPK-ACC signaling pathway might be associated with liver lipid deposition. Both chronic and acute exercises could increase the ACC phosphorylation. Chronic exercise can effectively repair the damage of the liver leptin-AMPK-ACC signaling pathway. Therefore, in the DCE group, liver glycogen and the leptin and insulin sensitivity were significantly increased. Moreover, TG and FFA decreased, which helped lowering both blood lipids and blood glucose.

In the DAE group, the leptin-AMPK-ACC signaling pathway was still active $8-16$ hours after acute exercise, which increased insulin sensitivity, but had no significant effect on liver glycolipid storage.

\section{Discussion}

Our results indicate that a long-term high fat diet plus low dose of STZ could induce disorders of the whole system and of liver lipids in middle-aged rats, which are associated with both insulin and leptin resistance. These phenomena are similar to the onset, progression, and metabolic characteristics of type 2 diabetes in humans [3-6]. The interruption of the liver leptin-AMPK-ACC signaling pathway might be one of the glucose and lipid metabolism disorders found in type 2 diabetes. Eight weeks of chronic exercise not only effectively improved the leptin-AMPK-ACC signaling pathway, but also alleviated the liver and whole system lipid disorders and partially reversed leptin and insulin resistance. Acute exercise could activate the leptin-AMPK-ACC signaling pathway and reduce the blood glucose level for at least $8-16$ hours but has no significant effect on hepatic glucose and lipid metabolism.

It has been widely accepted that excessive fat accumulation is strongly correlated with the insulin resistance and leptin resistance in peripheral tissues (muscle and liver) [14, 30-35]. Excessive nutritional and lipid deposition could lead to an increase in the number of fat cells and further stimulates the leptin secretion. Once leptin binds its receptor, triglyceride synthesis will be inhibited, which further promotes the oxidation of free fatty acids in order to avoid excessive lipid deposition [36] and maintain lean body mass [37]. Some studies have suggested that skeletal muscle leptin can activate the AMPK pathway in two ways. In the first activation, leptin acts directly with skeletal muscle to induce the rapid and transient activation of AMPK, and in the second activation mechanism, one is the $\alpha$ adrenal system activating AMPK through the hypothalamus sympathetic skeletal muscle and inducing ACC phosphorylation, which further reduces the synthesis of fatty acids [7]. This activated Malonyl-CoA decarboxylase pathway reduces the level of Malonyl-CoA (MA), inhibits the synthesis of fat, eliminates the inhibition of carnitine palmitoyltransferase 1 (CTP1) [38], promotes long-chain fatty acids in the mitochondrial inner membrane, and increases fat oxidation and decomposition [8, 39, 40]. Lacking leptin or the leptin receptor in diabetic rats ( $\mathrm{fa} / \mathrm{fa}$ and $\mathrm{ZDF}$ ) reduces the AMPK activity in skeletal muscle and in liver, and promotes fat storage. Administration of leptin or AMPK activators can effectively prevent the development of diabetes [41]. However, the effects on the liver leptin-AMPKACC pathway of diabetic rats induced by high fat diet plus low dose of STZ are unclear.

Our results indicate that in the DC group, expression of liver leptin significantly increased, but expression of leptin receptor decreased. Moreover, expression and phosphorylation of AMPK $\alpha 1 / 2, A M P K \alpha 1$, and AMPK $\alpha 2$ were effectively inhibited. The ACC phosphorylation was also inhibited, which was associated with a decline of glycogen and an increase of TG and FFA. This data suggests that the liver leptin-AMPK-ACC signaling pathway is related to hepatic 

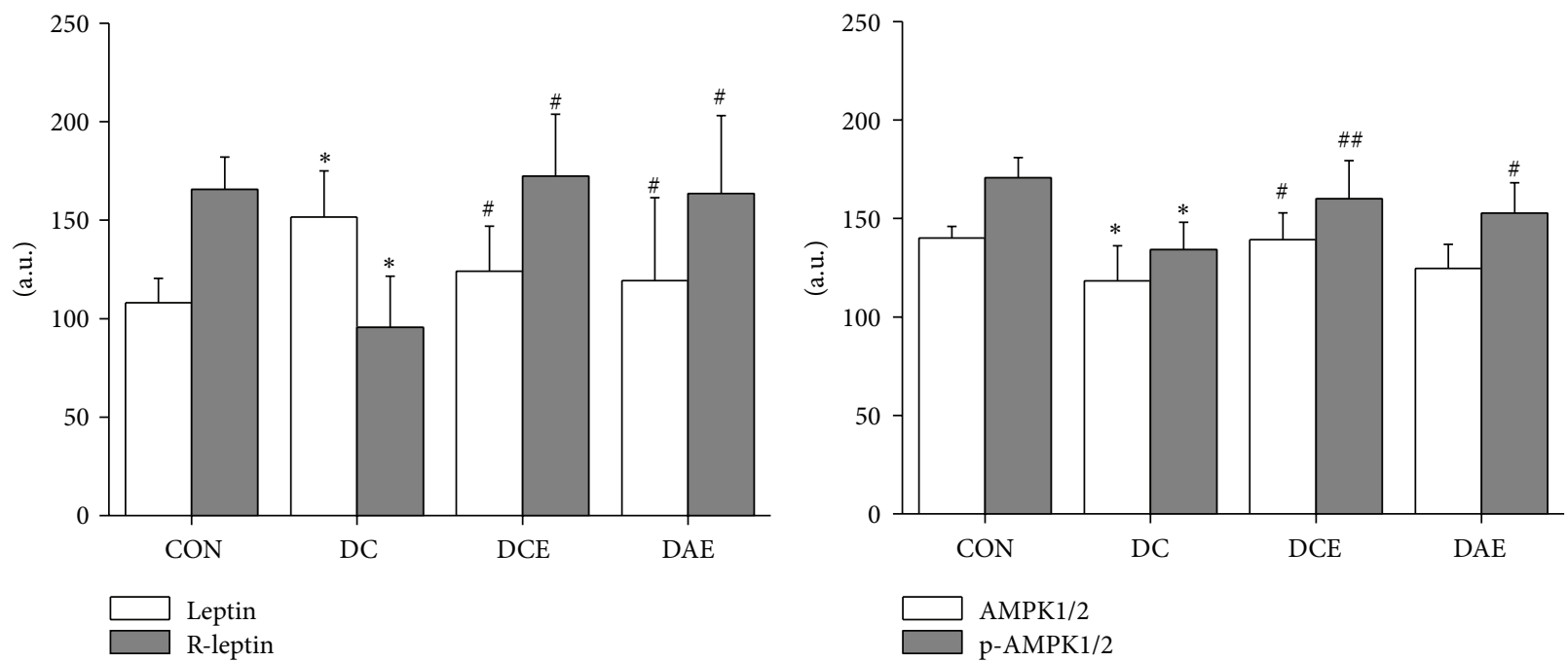

(a)
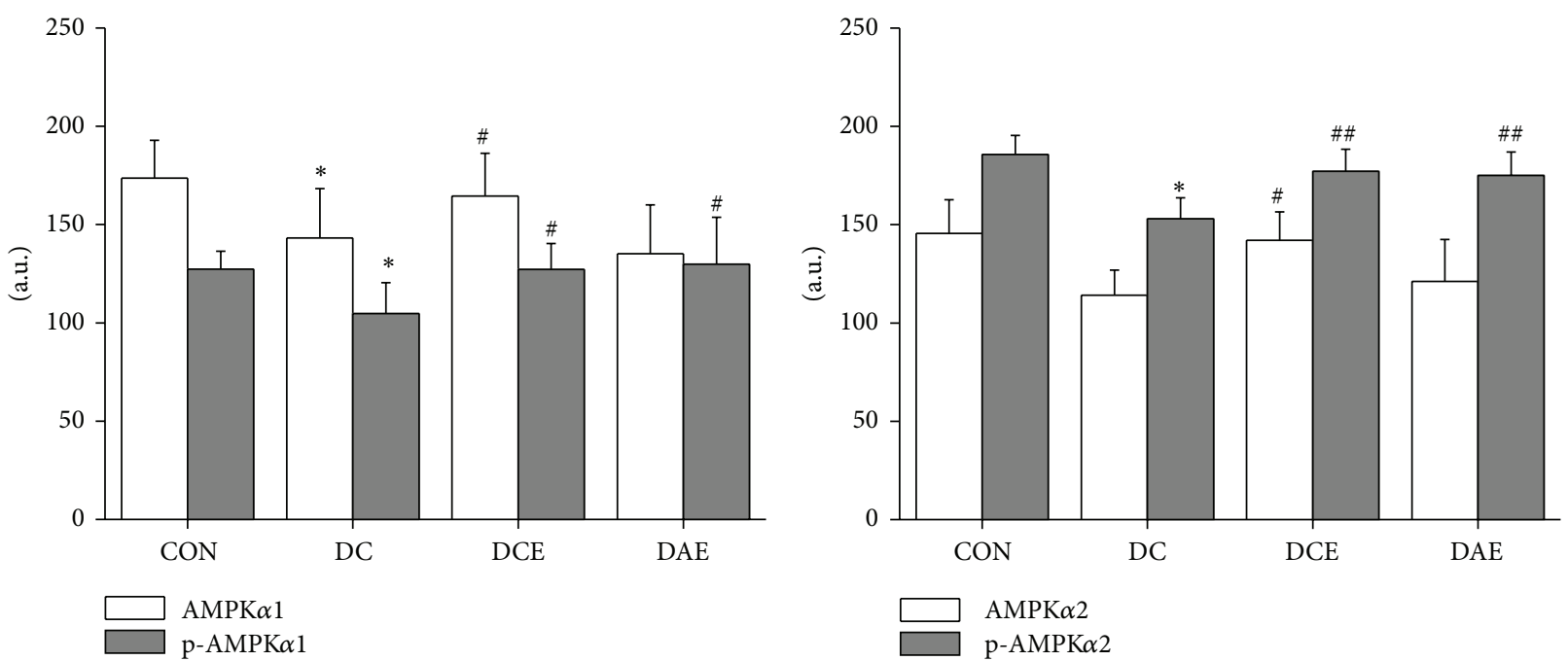

(c)

(d)

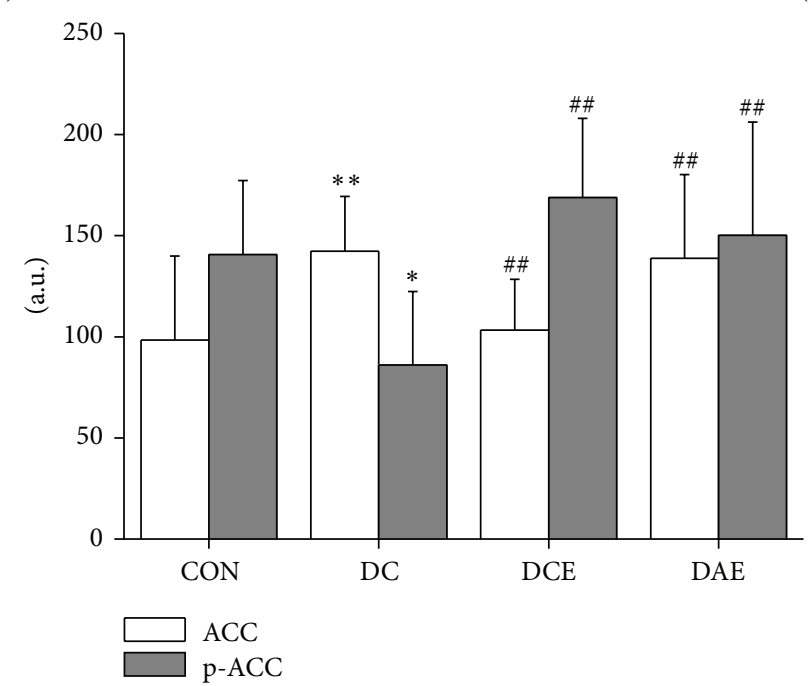

(e)

FIGURE 1: Histograph showing the effects of exercise on the leptin-AMPK-ACC pathway in type 2 diabetes rats. The relative levels of protein phosphorylation and protein expression related to the leptin-AMPK-ACC signaling pathway on different groups were shown. (a) Leptin and its receptor. (b) AMPK1/2 and p-AMPK1/2 $\left(\mathrm{Thr}^{172}\right.$ ). (c) AMPK $\alpha 1$ and p-AMPK $\alpha 1\left(\mathrm{Thr}^{172}\right.$ ). (d) AMPK $\alpha 2$ and p-AMPK $\alpha 2\left(\mathrm{Thr}^{172}\right)$. (e) ACC and p-ACC $\left(\mathrm{Ser}^{79}\right)$. Note: in comparison with the control group: ${ }^{* *} P<0.01,{ }^{*} P<0.05$; in comparison with the diabetes group: ${ }^{\#} P<0.01$, ${ }^{\#} P<0.05(n=8)$. 


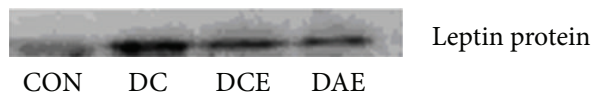

(a)

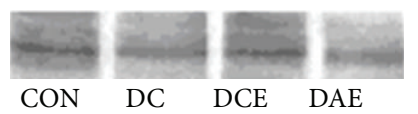

AMPK $\alpha$ 1/2 protein

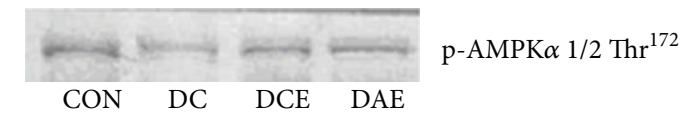

(b)

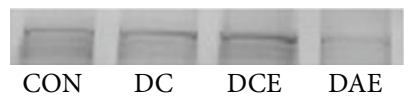

AMPK $\alpha 1$ protein

(c)

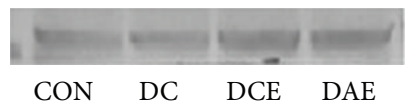

AMPK $\alpha 2$ protein

(d)

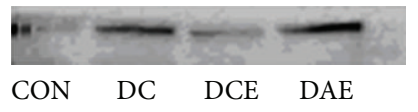

ACC protein

(e)

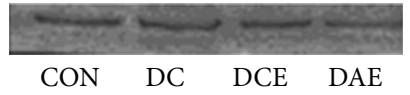

GAPDH

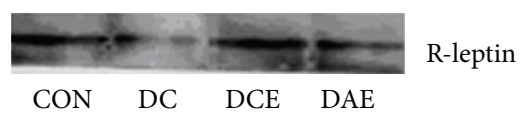

CON DC DCE DAE
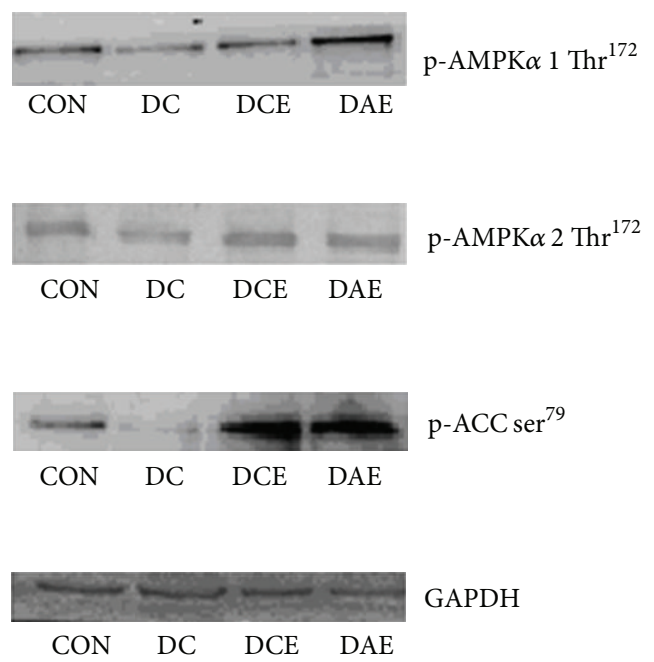

(f)

FIGURE 2: Western blotting images of protein expression and protein phosphorylation related to the leptin-AMPK-ACC signaling pathway of different groups of type 2 diabetes rats. (a) Leptin and its receptor. (b) AMPK $\alpha 1 / 2$ and p-AMPK $\alpha 1 / 2$. (c) AMPK $\alpha 1$ and p-AMPK $\alpha 1$. (d) AMPK $\alpha 2$ and p-AMPK $\alpha 2$. (e) ACC and p-ACC. (f) GADPH as an internal standard.

glucose and lipid metabolic disorder and might explain the etiology of fatty liver incidence in patients with diabetes.

Changes in body weight may play a role in the prevention of hyperglycemia caused by regular physical activity, although it is not likely to be the only explanation. In our study, diabetic trained animals showed a slight increase in weight, which puts in doubt whether improvements in insulin sensitivity are consequences of weight gain effects. For this purpose, blood triglyceride, total cholesterol, and free fatty acid concentrations were also examined and found to be remarkably reduced by exercise training. This relative decrease in biochemical parameters in diabetic trained animals may have contributed, at least in part, to their improved insulin sensitivity. Furthermore, diabetic animals were submitted to acute exercise, which had no effect on body weight. While the impact of chronic exercise on leptin was related to physical condition, chronic exercise does not have any effect on the blood leptin levels of athletes but reduces the leptin levels of nonathletes with normal weight $[42,43]$, obese individuals, and obese rats and also reduces body fat and accumulation of skeletal muscle lipids, which can prevent or mitigate leptin resistance [44]. Appropriate exercise can effectively reduce serum leptin of diabetic rats and humans [45], ease leptin resistance, and inhibit the development of diabetes. The impact of chronic exercise on the leptin receptor is also controversial. Research indicates that chronic exercise could downregulate leptin receptor gene expression in the hypothalamus [46] and improve the insulin resistance of aging rats. Another study suggested that chronic exercise could reduce the expression of the liver leptin receptor gene and decrease plasma leptin levels in rats with a high fat diet [47]. In our previous study, we found that chronic exercise could reduce body fat and blood leptin levels but elevate gene expression of the leptin receptor in the adipose tissue of obese rats as well as improve leptin resistance. In agreement with our previous study, decreased leptin expression and increased expression of the leptin receptor were also found in the high fat diet plus low dose STZ-induced type 2 diabetic rats, 24-36 hours after chronic exercise. In agreement with published data, endurance exercise could also increase gene expression of the hypothalamic leptin receptor and activation of the JAK2STAT3 signaling pathway, reducing leptin and insulin levels [48]. Increased expression of the leptin receptor was found in hypertrophied triceps of professional tennis players [49].

The impact of chronic exercise on the AMPK pathway was mostly studied in skeletal muscle. Chronic exercise can effectively reduce ceramide levels in the skeletal muscle of the high fat diet rats and restore insulin-stimulated glucose transport in skeletal muscle. Moreover, leptin stimulated phosphorylation in the skeletal muscle AMPK-ACC pathway, 
and promoted the oxidative decomposition of the FA [44, 50]. In Zucker and OLETF rats, where both lines have metabolic abnormalities, chronic exercise induced increased phosphorylation of ACC, and fat decomposition, as well as reduced fat synthesis in skeletal muscle. The impact of chronic exercise on the liver leptin-AMPK-ACC pathway has not been reported, although increase of liver ACC phosphorylation and AMPK $\alpha 1-\alpha 2$-subunit mRNA/protein expression has been found [51]. In our present study, we found that not only was liver AMPK $\alpha 1 / 2$, AMPK $\alpha 1$, and AMPK $\alpha 2$ protein expression increased, but increased phosphorylation levels were also seen, which further induced ACC phosphorylation, inhibited TG synthesis, promoted FFA oxidation, reduced lipid storage in the liver, and abolished insulin and leptin resistance. The impact of acute exercise on leptin could be influenced by many factors such as exercise stress and intensity, as well as the physical condition of the individual. Bouassida reported that the effects of one-time exercise on leptin levels were related to energy consumption and exercise time. Energy consumption less than $800 \mathrm{kcal}$ or $<60 \mathrm{~min}$ of active movement does not change the level of serum leptin; however, energy consumption greater than $800 \mathrm{kcal}$ or $\geq 60 \mathrm{~min}$ could stimulate the lipolysis and reduce serum leptin level [13]. 8-16 hours after acute exercise (90 min $\times 2$ ), leptin expression was significantly decreased and its receptor expression was significantly increased in the liver of diabetic rats. Moreover, AMPK $\alpha 1 / 2$, AMPK $\alpha 1$, AMPK $\alpha 2$ and ACC phosphorylation levels increase, but no significant effects are noticeable on liver lipid and glycogen storage. Our data suggested that acute exercise could activate the liver leptin-AMPK-ACC signaling pathway, promote lipid mobilization, and inhibit lipid synthesis. This result has also been shown in other tissues. In agreement with our previous studies, AMPK $\alpha \operatorname{Thr}^{172}$ and the ACC phosphorylation levels were increased in skeletal muscle after 3 hours of acute exercise [23]. Enhanced skeletal muscle AMPK $\alpha 2$ activity, AMPK $\alpha 2$, and ACC phosphorylation levels were also found in similar human studies [52, 53]. Moreover, Koh et al. further confirmed that acute treadmill exercise reduces ACC adipocyte activity in rats [54].

\section{Summary}

Impaired liver leptin-AMPK-ACC signaling pathways were closely related to glucose and lipid metabolism disorders in high fat diet plus low dose of STZ-induced type 2 diabetic rats. We further confirmed that chronic exercise could indirectly repair the leptin-AMPK-ACC signaling pathway in these rats, alleviate liver and body lipid disorders, and improve the IR and LR. Acute exercise could also indirectly activate the liver leptin-AMPK-ACC signaling pathway and increase insulin sensitivity, but it should be noted that irreversible liver lipid disorders are induced by a high fat diet.

\section{Conflict of Interests}

The authors declare that they have no conflict of interests.

\section{Acknowledgments}

The authors thank the National Natural Science Foundation of China for the financial support (Grant nos. 30971414 and 81273096) and they also thank Li Bowen, Zhao Gang, and Liu Huili, teachers of China Medical University, for their help with experimental processing. Secondly, the authors owe thanks to graduate Song Chao, $\mathrm{Li} \mathrm{Yu}$, and Fan Changming for the assistance with model establishment. Thirdly, they want to give their thanks to the staff of Key Laboratory of Shenyang Sport University for providing them with the equipment.

\section{References}

[1] K. F. Petersen, E. A. Oral, S. Dufour et al., "Leptin reverses insulin resistance and hepatic steatosis in patients with severe lipodystrophy," Journal of Clinical Investigation, vol. 109, no. 10, pp. 1345-1350, 2002.

[2] E. A. Oral, V. Simha, E. Ruiz et al., "Leptin-replacement therapy for lipodystrophy," The New England Journal of Medicine, vol. 346 , no. 8, pp. 570-578, 2002.

[3] J. M. Friedman, "Modern science versus the stigma of obesity," Nature Medicine, vol. 10, no. 6, pp. 563-569, 2004.

[4] M. Mapfei, J. Halaas, E. Ravussin et al., "Leptin levels in human and rodent: measurement of plasma leptin and ob RNA in obese and weight-reduced subjects," Nature Medicine, vol. 1, no. 11, pp. 1155-1161, 1995.

[5] A. Widjaja, I. M. Stratton, R. Horn, R. R. Holman, R. Turner, and G. Brabant, "UKPDS 20: plasma leptin, obesity, and plasma insulin in type 2 diabetic subjects," Journal of Clinical Endocrinology and Metabolism, vol. 82, no. 2, pp. 654-657, 1997.

[6] S. B. Heymsfield, A. S. Greenberg, K. Fujioka et al., "Recombinant leptin for weight loss in obese and lean adults: a randomized, controlled, dose-escalation trial," Journal of the American Medical Association, vol. 282, no. 16, pp. 1568-1575, 1999.

[7] A. Sriwijitkamol, D. K. Coletta, E. Wajcberg et al., "Effect of acute exercise on AMPK signaling in skeletal muscle of subjects with type 2 diabetes: a time-course and dose-response study," Diabetes, vol. 56, no. 3, pp. 836-848, 2007.

[8] A. E. Jeukendrup, "Regulation of fat metabolism in skeletal muscle," Annals of the New York Academy of Sciences, vol. 967, pp. 217-235, 2002.

[9] K. L. Mullen, J. Pritchard, I. Ritchie et al., "Adiponectin resistance precedes the accumulation of skeletal muscle lipids and insulin resistance in high-fat-fed rats," American Journal of Physiology-Regulatory Integrative and Comparative Physiology, vol. 296, no. 2, pp. R243-R251, 2009.

[10] X. J. Yi, H. Wang, and Q. P. Li, "Exercise on the sexual development and the fat leptin receptor mRNA expression in the high-fat die female rats," Zhongguo Ying Yong Sheng Li Xue Za Zhi, vol. 25, no. 4, pp. 454-542, 2009.

[11] T. Fuentes, I. Ara, A. Guadalupe-Grau et al., "Leptin receptor $170 \mathrm{kDa}$ (OB-R170) protein expression is reduced in obese human skeletal muscle: a potential mechanism of leptin resistance," Experimental Physiology, vol. 95, no. 1, pp. 160-171, 2010.

[12] G. R. Steinberg, D. J. Dyck, J. Calles-Escandon et al., "Chronic leptin administration decreases fatty acid uptake and fatty acid transporters in rat skeletal muscle," Journal of Biological Chemistry, vol. 277, no. 11, pp. 8854-8860, 2002. 
[13] G. K. Bandyopadhyay, J. G. Yu, J. Ofrecio, and J. M. Olefsky, "Increased malonyl-CoA levels in muscle from obese and type 2 diabetic subjects lead to decreased fatty acid oxidation and increased lipogenesis; thiazolidinedione treatment reverses these defects," Diabetes, vol. 55, no. 8, pp. 2277-2285, 2006.

[14] B. Vozarova, N. Stefan, R. S. Lindsay et al., "High alanine aminotransferase is associated with decreased hepatic insulin sensitivity and predicts the development of type 2 diabetes," Diabetes, vol. 51, no. 6, pp. 1889-1895, 2002.

[15] D. J. Chiang, M. T. Pritchard, and L. E. Nagy, "Obesity, diabetes mellitus, and liver fibrosis," American Journal of PhysiologyGastrointestinal and Liver Physiology, vol. 300, no. 5, pp. G697G702, 2011.

[16] M. Lazo and J. M. Clark, "The epidemiology of nonalcoholic fatty liver disease: a global perspective," Seminars in Liver Disease, vol. 28, no. 4, pp. 339-350, 2008.

[17] R. Lautamäki, R. Borra, P. Iozzo et al., "Liver steatosis coexists with myocardial insulin resistance and coronary dysfunction in patients with type 2 diabetes," American Journal of PhysiologyEndocrinology and Metabolism, vol. 291, no. 2, pp. E282-E290, 2006.

[18] K. A. Krawczewski Carhuatanta, G. Demuro, M. H. Tschöp, P. T. Pfluger, S. C. Benoit, and S. Obici, "Voluntary exercise improves high-fat diet-induced leptin resistance independent of adiposity," Endocrinology, vol. 152, no. 7, pp. 2655-2664, 2011.

[19] R. Sari, M. K. Balci, N. Balci, and U. Karayalcin, "Acute effect of exercise on plasma leptin level and insulin resistance in obese women with stable caloric intake," Endocrine Research, vol. 32, no. 1-2, pp. 9-17, 2007.

[20] J. Zhao, Y. Tian, J. Xu, D. Liu, X. Wang, and B. Zhao, "Endurance exercise is a leptin signaling mimetic in hypothalamus of Wistar rats," Lipids in Health and Disease, vol. 10, article 225, 2011.

[21] C. M. Patterson, S. G. Bouret, A. A. Dunn-Meynell, and B. E. Levin, "Three weeks of postweaning exercise in DIO rats produces prolonged increases in central leptin sensitivity and signaling," American Journal of Physiology-Regulatory Integrative and Comparative Physiology, vol. 296, no. 3, pp. R537-R548, 2009.

[22] N. B. Ruderman, H. Park, V. K. Kaushik et al., "AMPK as a metabolic switch in rat muscle, liver and adipose tissue after exercise," Acta Physiologica Scandinavica, vol. 178, no. 4, pp. 435-442, 2003.

[23] S. Cao, B. Li, X. Yi et al., "Effects of exercise on AMPK signaling and downstream components to PI3K in rat with type 2 diabetes," PLoS ONE, vol. 7, no. 12, Article ID e51709, 2012.

[24] K. Srinivasan, B. Viswanad, L. Asrat, C. L. Kaul, and P. Ramarao, "Combination of high-fat diet-fed and low-dose streptozotocintreated rat: a model for type 2 diabetes and pharmacological screening," Pharmacological Research, vol. 52, no. 4, pp. 313-320, 2005.

[25] M. Zhang, X.-Y. Lv, J. Li, Z.-G. Xu, and L. Chen, “The characterization of high-fat diet and multiple low-dose streptozotocin induced type 2 diabetes rat model," Experimental Diabetes Research, vol. 2008, Article ID 704045, 9 pages, 2008.

[26] E. Luciano, E. M. Carneiro, C. R. Carvalho et al., "Endurance training improves responsiveness to insulin and modulates insulin signal transduction through the phosphatidylinositol 3kinase/Akt-1 pathway," European Journal of Endocrinology, vol. 147, no. 1, pp. 149-157, 2002.

[27] A. V. Chibalin, M. Yu, J. W. Ryder et al., "Exercise-induced changes in expression and activity of proteins involved in insulin signal transduction in skeletal muscle: differential effects on insulin-receptor substrates 1 and 2," Proceedings of the National Academy of Sciences of the United States of America, vol. 97, no. 1, pp. 38-43, 2000.

[28] U. K. Laemmli, "Cleavage of structural proteins during the assembly of the head of bacteriophage T4," Nature, vol. 227, no. 5259, pp. 680-685, 1970.

[29] S. Tanaka, T. Hayashi, T. Toyoda et al., "High-fat diet impairs the effects of a single bout of endurance exercise on glucose transport and insulin sensitivity in rat skeletal muscle," Metabolism, vol. 56, no. 12, pp. 1719-1728, 2007.

[30] K. F. Petersen and G. I. Shulman, "Etiology of insulin resistance," American Journal of Medicine, vol. 119, no. 5, pp. S10-S16, 2006.

[31] P.-J. Hsiao, K.-K. Kuo, S.-J. Shin et al., "Significant correlations between severe fatty liver and risk factors for metabolic syndrome," Journal of Gastroenterology and Hepatology, vol. 22, no. 12, pp. 2118-2123, 2007.

[32] G. Targher and G. Arcaro, "Non-alcoholic fatty liver disease and increased risk of cardiovascular disease," Atherosclerosis, vol. 191, no. 2, pp. 235-240, 2007.

[33] E. Fabbrini, S. Sullivan, and S. Klein, "Obesity and nonalcoholic fatty liver disease: biochemical, metabolic, and clinical implications," Hepatology, vol. 51, no. 2, pp. 679-689, 2010.

[34] E. K. Speliotes, J. M. Massaro, U. Hoffmann et al., "Fatty liver is associated with dyslipidemia and dysglycemia independent of visceral fat: the Framingham heart study," Hepatology, vol. 51, no. 6, pp. 1979-1987, 2010.

[35] E. Fabbrini, F. Magkos, B. S. Mohammed et al., "Intrahepatic fat, not visceral fat, is linked with metabolic complications of obesity," Proceedings of the National Academy of Sciences of the United States of America, vol. 106, no. 36, pp. 15430-15435, 2009.

[36] M. Shimabukuro, K. Koyama, G. Chen et al., "Direct antidiabetic effect of leptin through triglyceride depletion of tissues," Proceedings of the National Academy of Sciences of the United States of America, vol. 94, no. 9, pp. 4637-4641, 1997.

[37] R. H. Unger, "The Physiology of Cellular Liporegulation," Annual Review of Physiology, vol. 65, pp. 333-347, 2003.

[38] M. Palou, T. Priego, J. Sánchez et al., "Sequential changes in the expression of genes involved in lipid metabolism in adipose tissue and liver in response to fasting," Pflugers Archiv European Journal of Physiology, vol. 456, no. 5, pp. 825-836, 2008.

[39] Y. Minokoshi, T. Alquier, H. Furukawa et al., "AMP-kinase regulates food intake by responding to hormonal and nutrient signals in the hypothalamus," Nature, vol. 428, no. 6982, pp. 569-574, 2004.

[40] L. Miyamoto, K. Ebihara, T. Kusakabe et al., "Leptin activates hepatic $5^{\prime}$-AMP-activated protein kinase through sympathetic nervous system and ?1-adrenergic receptor:a potential mechanism for improvement of fatty liver in lipodystrophy by leptin," The Journal of Biological Chemistry, vol. 287, no. 48, pp. 4044140447, 2012.

[41] X. Yu, S. McCorkle, M. Wang et al., "Leptinomimetic effects of the AMP kinase activator AICAR in leptin-resistant rats: prevention of diabetes and ectopic lipid deposition," Diabetologia, vol. 47, no. 11, pp. 2012-2021, 2004.

[42] K. A. Krawczewski Carhuatanta, G. Demuro, M. H. Tschöp, P. T. Pfluger, S. C. Benoit, and S. Obici, "Voluntary exercise improves high-fat diet-induced leptin resistance independent of adiposity," Endocrinology, vol. 152, no. 7, pp. 2655-2664, 2011.

[43] M.-S. Gauthier, K. Couturier, A. Charbonneau, and J.-M. Lavoie, "Effects of introducing physical training in the course 
of a 16-week high-fat diet regimen on hepatic steatosis, adipose tissue fat accumulation, and plasma lipid profile," International Journal of Obesity, vol. 28, no. 8, pp. 1064-1071, 2004.

[44] G. R. Steinberg, A. C. Smith, S. Wormald, P. Malenfant, C. Collier, and D. J. Dyck, "Endurance training partially reverses dietary-induced leptin resistance in rodent skeletal muscle," American Journal of Physiology-Endocrinology and Metabolism, vol. 286, no. 1, pp. E57-E63, 2004.

[45] L. Roselli-Rehfuss, K. G. Mountjoy, L. S. Robbins et al., "Identification of a receptor for $\gamma$ melanotropin and other proopiomelanocortin peptides in the hypothalamus and limbic system," Proceedings of the National Academy of Sciences of the United States of America, vol. 90, no. 19, pp. 8856-8860, 1993.

[46] G. R. Steinberg, A. C. Smith, S. Wormald, P. Malenfant, C. Collier, and D. J. Dyck, "Endurance training partially reverses dietary-induced leptin resistance in rodent skeletal muscle," American Journal of Physiology-Endocrinology and Metabolism, vol. 286, no. 1, pp. E57-E63, 2004.

[47] S. Yasari, D. Wang, D. Prud'homme, M. Jankowski, J. Gutkowska, and J.-M. Lavoie, "Exercise training decreases plasma leptin levels and the expression of hepatic leptin receptor-a, -b, and, -e in rats," Molecular and Cellular Biochemistry, vol. 324, no. 1-2, pp. 13-20, 2009.

[48] J. Zhao, Y. Tian, J. Xu, D. Liu, X. Wang, and B. Zhao, "Endurance exercise is a leptin signaling mimetic in hypothalamus of Wistar rats," Lipids in Health and Disease, vol. 10, article 225, 2011.

[49] H. Olmedillas, J. Sanchis-Moysi, T. Fuentes et al., "Muscle hypertrophy and increased expression of leptin receptors in the musculus triceps brachii of the dominant arm in professional tennis players," European Journal of Applied Physiology, vol. 108, no. 4, pp. 749-758, 2010.

[50] I. R. W. Ritchie, R. A. Gulli, L. E. Stefanyk, E. Harasim, A. Chabowski, and D. J. Dyck, "Restoration of skeletal muscle leptin response does not precede the exercise-induced recovery of insulin-stimulated glucose uptake in high-fat-fed rats," American Journal of Physiology-Regulatory Integrative and Comparative Physiology, vol. 300, no. 2, pp. R492-R500, 2011.

[51] K. Takekoshi, M. Fukuhara, Z. Quin et al., "Long-term exercise stimulates adenosine monophosphate-activated protein kinase activity and subunit expression in rat visceral adipose tissue and liver," Metabolism, vol. 55, no. 8, pp. 1122-1128, 2006.

[52] J. P. Little, A. Safdar, N. Cermak, M. A. Tarnopolsky, and M. J. Gibala, "Acute endurance exercise increases the nuclear abundance of PGC-1 $\alpha$ in trained human skeletal muscle," American Journal of Physiology-Regulatory Integrative and Comparative Physiology, vol. 298, no. 4, pp. R912-R917, 2010.

[53] R. S. Lee-Young, G. Koufogiannis, B. J. Canny, and G. K. McConell, "Acute exercise does not cause sustained elevations in AMPK signaling or expression," Medicine and Science in Sports and Exercise, vol. 40, no. 8, pp. 1490-1494, 2008.

[54] H.-J. Koh, M. F. Hirshman, H. He et al., "Adrenaline is a critical mediator of acute exercise-induced AMP-activated protein kinase activation in adipocytes," Biochemical Journal, vol. 403, no. 3, pp. 473-481, 2007. 


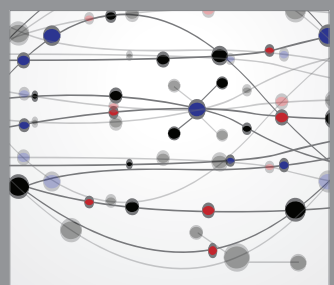

The Scientific World Journal
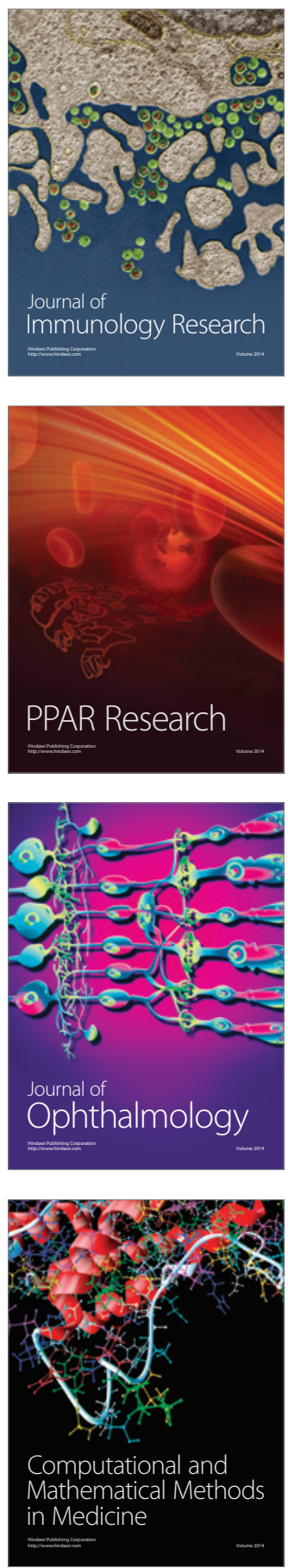

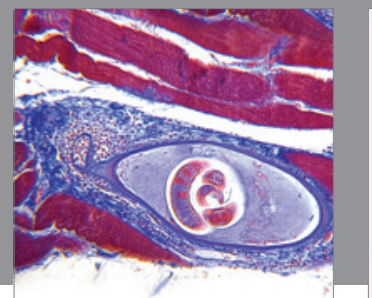

Gastroenterology

Research and Practice
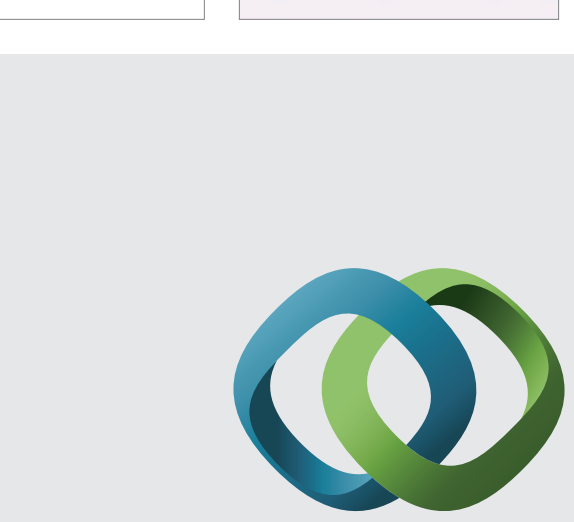

\section{Hindawi}

Submit your manuscripts at

http://www.hindawi.com
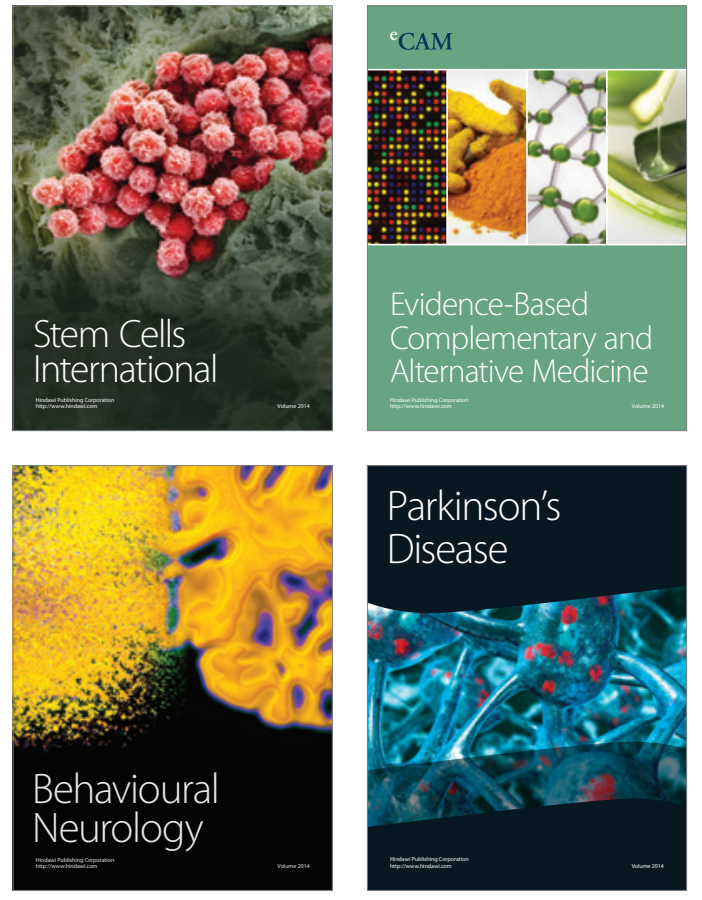
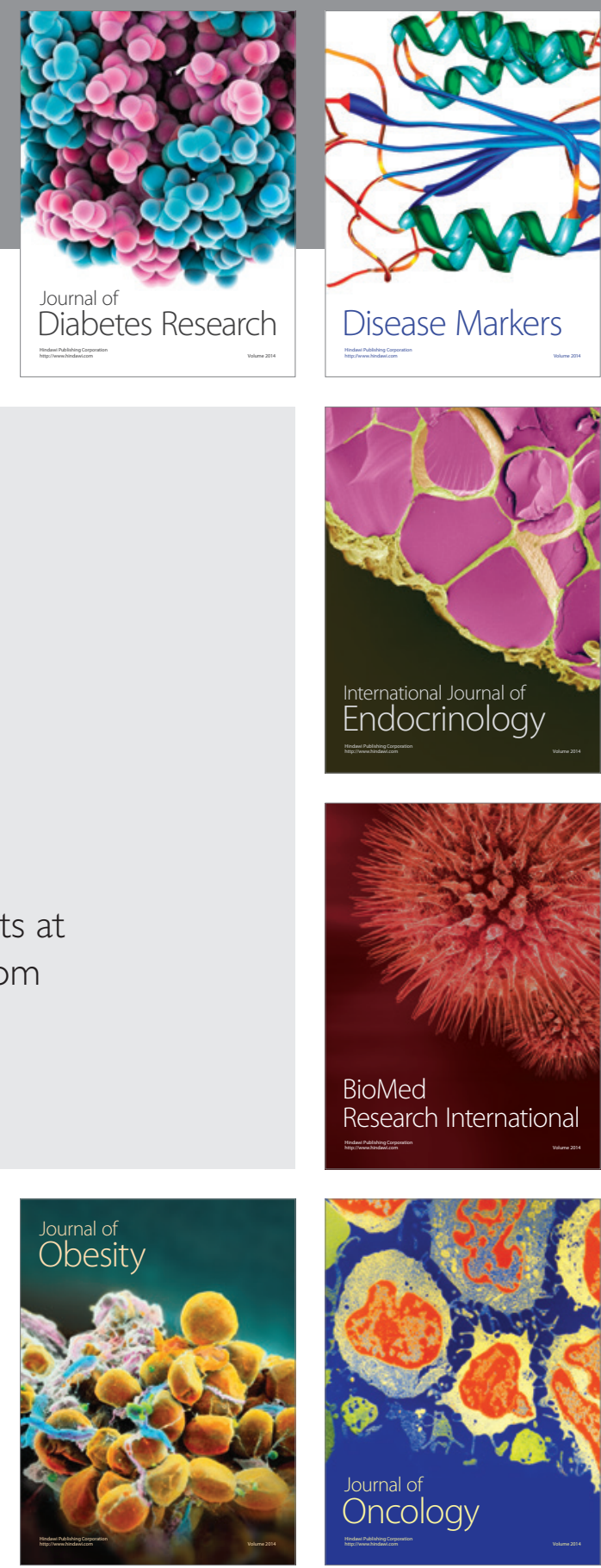

Disease Markers
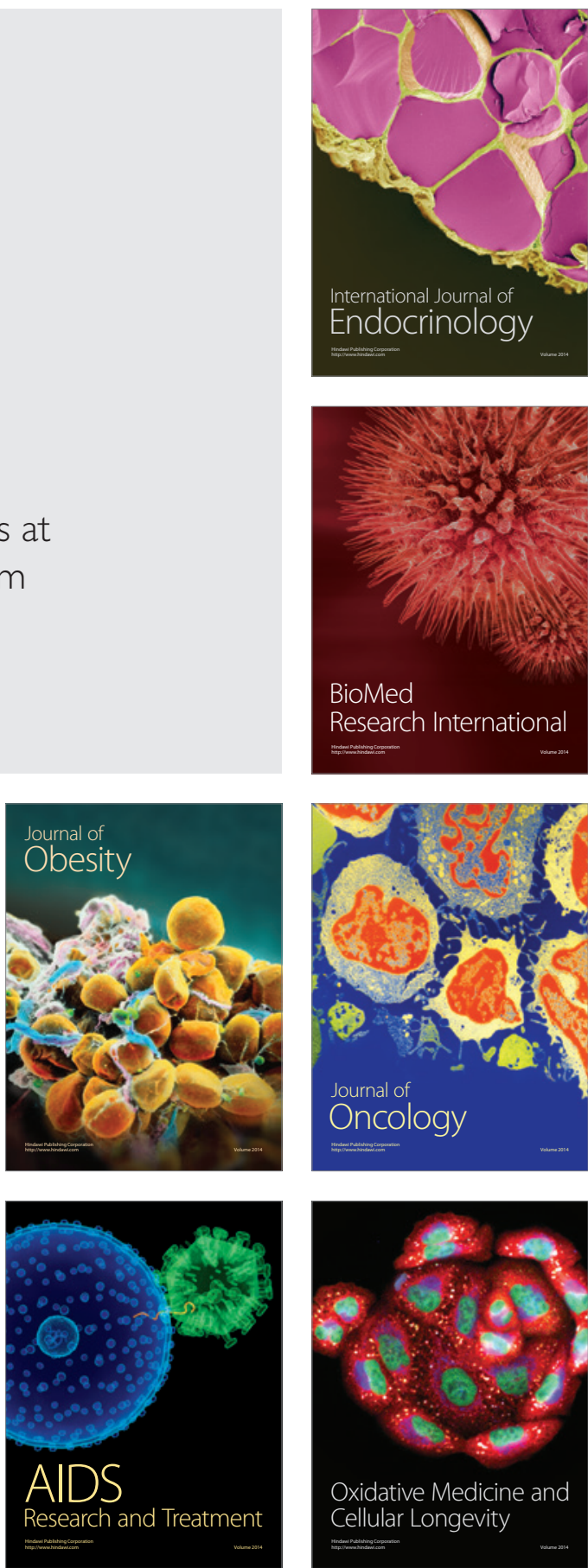